\title{
Computational Methodology for Iris Segmentation and Detection in Images from the Eyes Region Using Convolutional Neural Networks
}

\author{
Fredson Costa Rodrigues \\ Núcleo de Computação Aplicada \\ Universidade Federal do Maranhão \\ São Luís-MA, Brasil \\ fredsoncosta@nca.ufma.br
}

\author{
Geraldo Braz Júnior \\ Núcleo de Computação Aplicada \\ Universidade Federal do Maranhão \\ São Luís-MA, Brasil \\ geraldo@nca.ufma.br
}

\author{
Anselmo C. de Paiva \\ Núcleo de Computação Aplicada \\ Universidade Federal do Maranhão \\ São Luís-MA, Brasil \\ paiva@nca.ufma.br
}

\author{
Aristófanes Corrêa \\ Núcleo de Computação Aplicada \\ Universidade Federal do Maranhão \\ São Luís-MA, Brasil \\ ari@nca.ufma.br
}

\author{
João Dalysson S. de Almeida \\ Núcleo de Computação Aplicada \\ Universidade Federal do Maranhão \\ São Luís-MA, Brasil \\ jdallyson@nca.ufma.br
}

\author{
André Castelo Branco Soares \\ Departamento de Computação \\ Universidade Federal do Piauí \\ Teresina-PI, Brasil \\ andre.soares@ufpi.edu.br
}

\begin{abstract}
Eye tracking is an application of computer vision responsible for detecting the iris and pupil in the eye region. The usefulness of this tracking contributes to research that assesses cognitive aspects through pupillary reactions identified in these detected regions. Another application in this task is iris recognition in digital biometrics. This study aims to carry out the verification and detection of the iris in images of the eye region occluded by eyelashes, eyelids and specular reflexes, through a deep neural network called At-Unet in this article. In order to assist in eye tracking this method achieves $95.32 \%$ of data coefficient when segmenting the iris of the eyes, indicating the efficiency of this methodology.
\end{abstract}

Index Terms-Attention Module, At-Unet, Deep Neural Networks, íris segmentation, eye tracking.

\section{INTRODUÇÃO}

A detecção precisa da íris e pupila no rastreamento ocular é uma tarefa complexa. As condições de iluminação do ambiente, a forma de aquisição das imagens através de câmeras, e as pálpedras que geralmente cobrem o formato circular da íris e a pupila acabam comprometendo a detecção dessas regiões. A localização da região dos olhos e a segmentação da íris e pupila, são aplicações essênciais para um bom desempenho no rastreamento ocular.

A eficiência na etapa de segmentação da íris sofre com a oclusão parcial pelos cílios e pálpebras e pela ocorrência de brilhos especulares na região periocular, causando erros significativos na tarefa. Então é necessário remover os cílios, pálpebras e reflexos especulares para a extração da íris durante a segmentação, e dessa forma facilitar o seu reconhecimento.

Métodos de localização da íris baseados em aprendizado de máquina, histograma/limiar, Transformada de Hough e outras técnicas, tentam tornar o rastreamento ocular preciso diante de condições adversas [1].
A maioria dos métodos computacionais trabalham na localização da íris ou no seu reconhecimento [2], baseado no modelo de Daugman [3]. O modelo de reconhecimento da íris de Daugman apresenta três técnicas que podem ser, baseadas em contornos, na transformada de Fourier e inferências estatísticas para detectar e excluir cílios.

Abordagens baseadas em redes neurais convolucionais (CNN), para realizar a detecção da íris alcançam um novo patamar de desempenho comparado a métodos convencionais. As redes neurais convolucionais hierárquicas ( $\mathrm{HCNNs}$ ) e redes neurais de multi-escala, são exemplos de arquiteturas capazes de atingir a alta precisão na tarefa de segmentação da íris, de modo a obter bons resultados no rastreamento ocular [4].

Um outro trabalho relacionado e baseado em CNN's, é o FRED-Net [5]. É uma arquitetura de rede neural desenvolvida para a segmentação da íris, consistindo em uma rede residual de codificação e decodificação, na qual determina os limites externos da íris através de conexões de saltos residuais. Já o CNNHT [6], visa ajustar a parametrização da íris (ou seja a localização da íris), baseado na região segmentada gerada pela CNN. A arquitetura de rede neural Refinanet é usada juntamente com uma sequência de etapas de pós-processamento para ajustar os resultados da segmentação aos limites externos da íris. A proposta deste trabalho é auxiliar o rastreamento ocular, basedo em resultados precisos da segmentação da íris

O IrisParseNet [7] é uma rede neural de multitarefas para segmentar a região da íris e prever seus limites externos, possibilitando o uso de várias etapas de pós-processamento para detectar a íris. Desta forma, surgem constantemente novas abordagens para a segmentação da íris, no intuito de gerar novas aplicações no rastreamento ocular.

A fim de auxiliar psicólogos cognitvos, médicos e pesquisadores da visão computacional, no rastreamento ocular, 
este artigo propõe um método baseado em uma arquitetura de rede neural convolucional denominada de At-Unet. Esta arquitetura consiste na alteração da rede neural U-Net para a segmentação da íris em imagens da região dos olhos. Portanto a metodologia visa contribuir no rastreamento ocular, usando técnicas de redes neurais profundas para segmentar essa região e detectar os seus limites exteriores. A proposta é comparar este método com o estado da arte de segmentação e detecção da íris e assim validar a eficiência desta metodologia na tarefa.

Este artigo está organizado de modo que na próxima seção sejam apresentados os conceitos principais que embasam o trabalho desenvolvido, em seguida na seção 3 é apresentada a metodologia proposta. Na seção 4 apresentamos os resultados dos experimentos realizados para validar a metodologia e finalmente na última seção apresentamos as conclusões e sugestões de trabalhos futuros.

\section{FUndAMENTAÇÃO TEÓRICA}

Nesta seção, serão apresentadas os aspectos teóricos que fundamentam a metodologia neste trabalho. Serão abordados conceitos essênciais de arquiteturas de redes neurais convolucionais no propósito de destacar as principais características e contribuições na visão computacional.

\section{A. Redes Neurais Convolucionais}

As redes neurais fazem parte de um dos métodos de machine learning, e visam simular o funcionamento do cérebro humano baseado em várias funções matemáticas, contendo um conjunto de neurônios e suas ligações. O principal objetivo de uma rede neural é executar tarefas computacionais fundamentadas em ações cotidianas do ser humano, a exemplo a classificação de animais, pessoas e objetos. Esses tipos de atividades são complexas para uma máquina, e por esse motivo, técnicas baseadas em apredizado profundo são desenvolvidas para obter o melhor desempenho em tarefas da visão computacional.

Com o avanço nos campos de redes neurais profundas várias arquiteturas de redes neurais são propostas para pesquisas e produção de tecnologias com inteligência artificial. As redes neurais convolucionais (CNN) se constituem em uma dessas arquiteturas de redes neurais profundas, caracterizadas por uma organização de várias camadas convolucionais, camadas de ativação, de agrupamento e entre outras camadas em um único esqueleto [8].

As CNN's são poderosas e profundos algoritmos de aprendizagem, capazes de lidar com milhões de parâmetros, convolvendo as imagens $2 \mathrm{D}$ com filtros de vários tamanhos, extraindo e aprendendo máximo de características de uma imagem em uma determinada tarefa. [9].

A U-Net [10] é uma arquitetura de rede neural profunda para tarefas de segmentação de imagens baseada na classificação dos pixels. Essa arquitetura tem uma estrutura simétrica de codificador e decodificador com conexões de salto. $\mathrm{O}$ codificador contém uma sequência de camadas de convolução e camadas pooling, enquanto o decodificador realiza a expansão e usa convoluções transpostas ou deconvoluções [11].
Existem diversas adaptações e variações da arquitetura de rede U-Net apresentando um excelente desempenho em imagens biomédicas. A FD-Unet é uma readaptação da arquitetura, visando obter bons resultados de segmentação em imagens da região dos olhos. Essa alteração da arquitetura usa convolução dilatada em vez da convolução original, para extrair mais características globais das imagens processadas [12].

\section{Metodologia}

A metodologia proposta neste trabalho visa a utilização de uma arquitetura de rede neural U-Net para segmentar e detectar a íris em imagens da região dos olhos, oclusas por cílios, pálpebras, e reflexos especulares do ambiente. Neste trabalho a proposta original da arquitetura U-Net é modificada, através da substituição do codificador por uma arquitetura de rede neural VGG-16 [13] e o ajuste do decodificador original, incluindo módulos chamados de atenção. Sendo assim a arquitetura modificada é chamada de At-Unet.

Em seguida, as imagens segmentadas são pós-processadas com o algoritmo Smallest Circle e ajustadas para detecção da íris com sua consequente avaliação. A figura 1 apresenta as etapas da metodologia que serão detalhadas nas subseções a seguir.

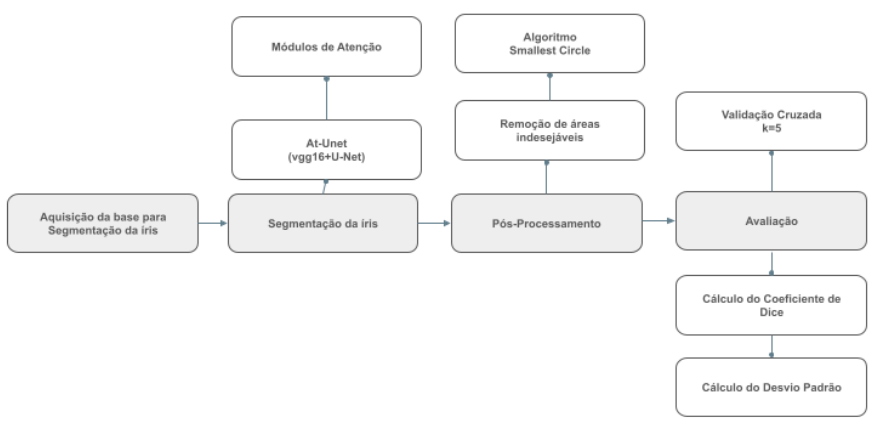

Fig. 1. Etapas da Metodologia.

A primeira etapa da metodologia é a aquisição de um conjunto de imagens contendo somente a região dos olhos. Em seguida é realizada a segmentação da íris utilizando a rede neural At-Unet. Após esta etapa são aplicados processos de pós-processamento para tratar artefatos que possam estar incluidos nesta região. Em seguida, a região da imagem segmentada e pós-processada é submetida ao processo de detecção, realizando o delineamento do círculo que define a íris. As subseções seguintes descrevem as principais etapas desta metodologia.

\section{A. Segmentação da Íris}

A segmentação automática da íris consiste em delimitar uma bounding box contendo a região da íris. Esta etapa é realizada através do uso de uma rede neural profunda denominada AtUnet. Essa rede neural é uma modificação na arquitetura UNet original. A modificação realizada consiste na utilização da arquitetura de rede VGG-16 como codificador da rede neural U-Net, e a inclusão dos módulos de atenção no decodificador. 
Na etapa de extração de características, durante a codificação da rede At-Unet, adota-se pesos pré-treinados do conjunto de dados Imagenet [14].

$\mathrm{Na}$ At-Unet, a VGG-16 consiste em 16 camadas divididas em cinco estágios que contém dois ou três camadas de convolução com filtros de tamanho 3x3, seguidas de normalização em lote e camadas de ativação Relu. A cada estágio de duas ou três camadas de convoluções, são adicionadas camadas de pooling com filtros de tamanho $2 \times 2$.

A decodificação da At-Unet é composta por etapas de upsampling para a expansão das dimensões dos mapas de características, por meio das conexões de saltos que concatenam os recursos extraídos do codificador com o decodificador, na intenção melhorar o aprendizado durante a expansão dos recursos concatenados. No decorrer da expansão dos mapas de caracterisitica, são implementadas os módulos de atenção. O módulo de atenção está antes da concatenação dos mapas de características do decodificar e o codificador, e entre as camadas de convolução transpostas. O objetivo do módulo de atenção é melhorar o poder de generalização da rede neural, dando uma ênfase maior em uma região de interesse durante o upsampling.

Antes de seguir o fluxo da rede neural, o módulo de atenção recebe dois vetores de características que irão passar por camadas de convolução 1x1 individuais, de modo que os dois vetores tenham as mesmas dimensões. Após essa operação os dois vetores, são somados elemento por elemento, e encaminhados a uma camada de ativação Relu e mais uma camada de convolução $1 \times 1$. O vetor resultante passa pela função de ativação sigmoide produzindo coeficientes de atenção(pesos), dando importâncias aos valores mais próximos de um. Em fim os coeficientes de atenção são ampliados para a dimensão do vetor original, usando uma interpolação trilinear, esses coeficientes são multiplicados com o vetor original elemento por elemento, escalando o vetor de acordo com os valores de importância definidas. Sendo assim o vetor resultante é transmitido normalmente na conexão de salto da rede neural At-Unet. Na figura 2 apresenta o módulo de atenção usado na arquitetura de rede neural.

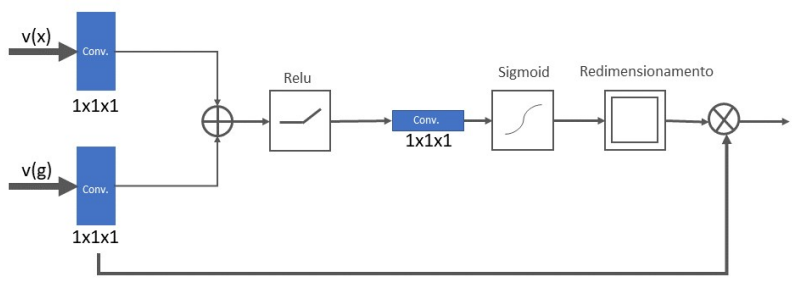

Fig. 2. Módulo de Atenção da Rede Neural At-Unet.

Em seguida são aplicadas operações de convoluções regulares, camadas de ativação relu e normalização em lote. No estágio final da decodificação são aplicados 32 filtros em uma sequência de camadas de convolução $3 \times 3$, seguidas de camadas Relu e normalização em lote, e por fim aplica-se uma camada de convolução $1 \times 1$ com 3 filtros, consequente da função de ativação sigmoide. A figura 3 apresenta a estrutura da arquitetura de rede de segmentação da íris At-Unet.

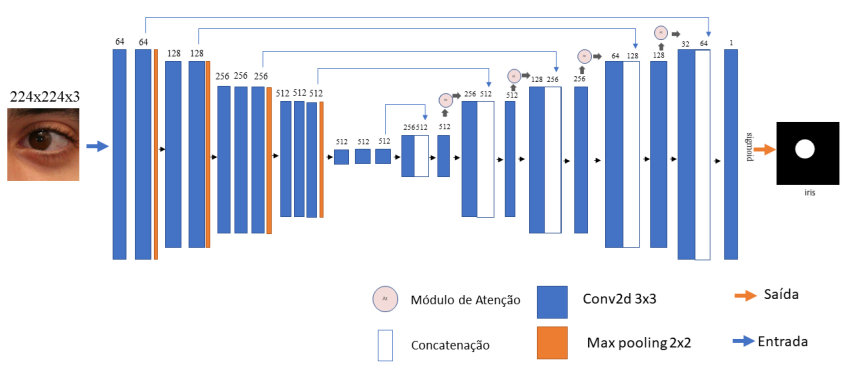

Fig. 3. Arquitetura da Rede Neural de Segmentação da íris At-Unet.

\section{B. Pós-processamento}

Foi verificada a necessidade de incluir uma etapa de pósprocessamento após a segmentaçào da íris. Esta etapa visa tratar alguns artefatos que se encontram nas regiões segmentadas e que não são do interesse do problema. A figura 4 exemplifica alguns desses artefatos.
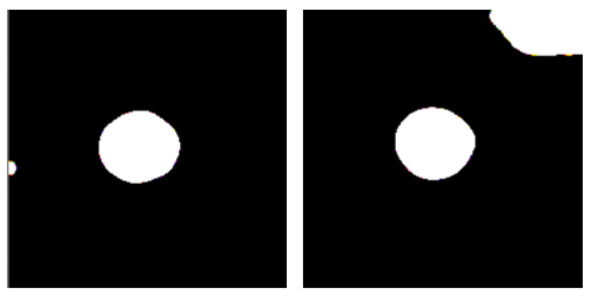

Fig. 4. Imagens segmentadas com áreas que não fazem parte da região da íris.

A etapa de pós-processamento visa remover essas áreas indesejáveis que podem surgir quando a região dos olhos é segmentada. São removidas as regiões com menor área dentre todas as regiões em uma mesma imagem. Este passo parte do princípio de que a íris é sempre a maior região segmentada na imagem.

Após essa etapa de remoção das áreas indesejáveis na imagem segmentada, é aplicado o algoritmo Smallest Circle, com o propósito de melhorar o delineamento da circuferência que representa a íris.

$\mathrm{O}$ algoritmo busca resolver um problema conhecido como problema dos círculos mínimos. Ele visa encontrar o menor círculo possível $D=[(x, y), r]$ em um conjunto de pontos de um plano euclidiano, onde $x$ e $y$ são as coordenadas do ponto e $r$ é o raio do círculo a ser criado. A proposta é gerar um círculo perfeito baseado nos pontos de pixels classificados corretamente como pertecendo à íris na saída da segmentação, corrigindo o formato da íris segmentada que pode estar deformada devido as oclusões causadas pelos cílios 
e pálpebras da região dos olhos. Na figura 5 há um exemplo da aplicação do algoritmo na imagem segmentada.

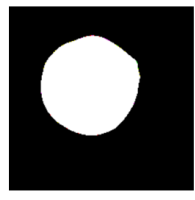

(a)

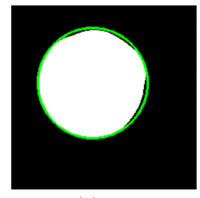

(b)

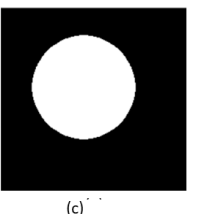

Fig. 5. Aplicação do algoritmo Smallest Circle. (a) Imagem resultante da Rede Neural de segmentação At-Unet com a remoção das regiões indesejáveis. (b) Imagem de exemplo da aplicação do algoritmo Smallest Circle para ajustar a íris segmentada. (c) Imagem resultante após a aplicação do algoritmo na íris.

Em seguida, ápos toda a etapa de segmentação da íris e pósprocessamento da imagem, é gerada um contorno em volta da região segmentada indicando os limites exteriores da íris, para opontar a detecção dessa região.

\section{EXPERIMENTOS E RESULTADOS}

Para validar a metodologia proposta são realizadas experimentos nas bases públicas, MICHE-I e UBIRIS.v2 [7]. As duas bases tem imagens da região dos olhos em quantidades diferentes, e disponibilizam as marcações Ground Truth da íris. A base MICHE-I contém 680 imagens e a base UBIRIS.v2 contém 483 imagens. Todas as imagens de treinamento, validação e teste da rede neural foram redimensionadas para um tamanho fixo de $224 \times 224$ pixels. A figura 5 apresenta exemplos dos dois conjuntos de dados usados neste trabalho.
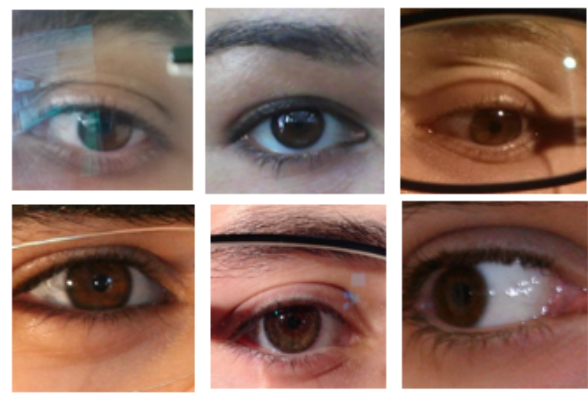

Fig. 6. Bases de dados MICHE-I e UBIRIS.v2.

Para validar o modelo de segmentação da íris, três experimentos são realizados. No primeiro experimento o treinamento da At-Unet é executada com o conjunto de dados MICHI-I. A base de 680 imagens, foi divida em $80 \%$ para treinamento, $10 \%$ para validação da rede neural e $10 \%$ para teste do modelo de segmentação. Durante o experimento é efetuado o aumento de dados, gerando para cada imagem da base de treinamento e validação, três novas images: rotacionadas em $90^{\circ}$, espelhadas verticalmente e espelhadas horizontalmente. Com este aumento de dados o conjunto de imagens totaliza em 1653 imagens de treinamento e 204 imagens para validação da rede neural.

O segundo experimento gera um modelo de segmentação da íris a partir do conjunto de dados UBIRIS.v2. As imagens da base foram divididas, $80 \%$ para treinamento da rede At-Unet, $10 \%$ para validação e $10 \%$ para teste do modelo. Durante o experimento foi utilizado o mesmo processo de aumento de dados nas imagens de treinamento e validação da rede conforme o experimento 1. Ao final, os dados de treino totalizam em 792 imagens e os dados de validação em 143 imagens.

O terceiro experimento é baseado na validação cruzada do modelo gerado na At-Unet a partir dos conjuntos de dados do experimento 1 e 2 . Durante a validação cruzada, as bases de dados foram divididas em cinco partes aleatórias ou seja $k=5$, ambas estão subdivididas em treinamento, validação e teste, conforme os experimentos 1 e 2 .

$\mathrm{Na}$ execução dos experimentos as configurações dos hiperparâmetros da rede neural são ajustadas para usar o método de otimização Adam, com a taxa de aprendizagem de 0.00001 e o decay de 0.000001. Além disso a função de perda de entropia cruzada é selecionada para verificar o desempenho da rede neural durante o treinamento em cem épocas. Em um certo ponto da execução do treinamento no decorrer dos experimentos, a perda de entropia cruzada deixa de convergir para o mínimo, ao longo de dez épocas, dessa forma o treinamento da rede neural é encerrada. Na figura 7 apresenta os gráficos da função de perda do experimento 1 e 2 .

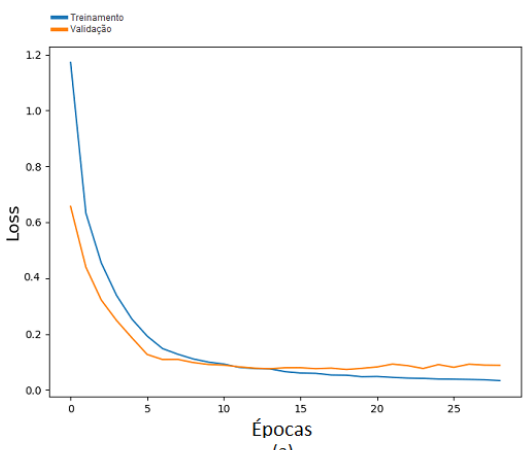

(a)

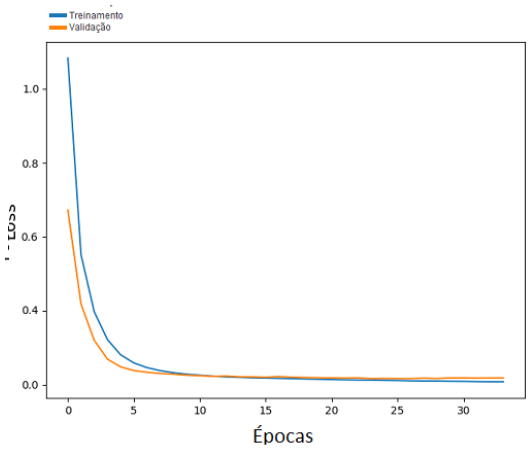

Fig. 7. Gráficos da função de Perda. (a) Gráfico do experimento 1. (b) Gráfico do experimento 2 .

O gráfico (a) representa o experimento 1, onde apresenta a constância na convergêcia da função de perda para os dados de valição do modelo até a época quinze. Após a esta época, 
a função de perda deixa de convergir para mínimo por pelo menos dez épocas seguidas, indicando que a partir deste ponto, o modelo não está generalizando os dados de treinamento. Portanto, o treinamento da rede neural convolucional é encerrada automaticamente.

O gráfico (b) do experimento 2, tem um comportamento diferente do gráfico (a). A função de perda deste treino, convergi para o mínimo até a época vinte, e em seguida se mantém estável por mais algumas épocas. Assim como o primeiro experimento, o treinamento é encerrado automaticamente, quando indentificada o aumento da função de perda durante um período de dez épocas.

Mesmo os gráficos apresentando comportamentos distintos, eles demonstram que os dados durante o treino, não estão sofrendo um sobreajuste para prejudicar o desempenho do modelo durante os testes.

Para avaliar o desempenho dos modelos ao longo dos experimentos usou-se o coeficiente de dice. $\mathrm{O}$ coeficiente de dice mede a sobreposição espacial entre duas imagens binárias. É usada para avaliar o desempenho da segmentação e seus valores que variam de 0 (sem sobreposição) a 1 (sobreposição perfeita) [15]. Todas as imagens de testes são diferentes das imagens de treinamento da rede neural, mas fazem parte da mesma base de dados. A figura 8 apresenta alguns resultados finais da metodologia completa.

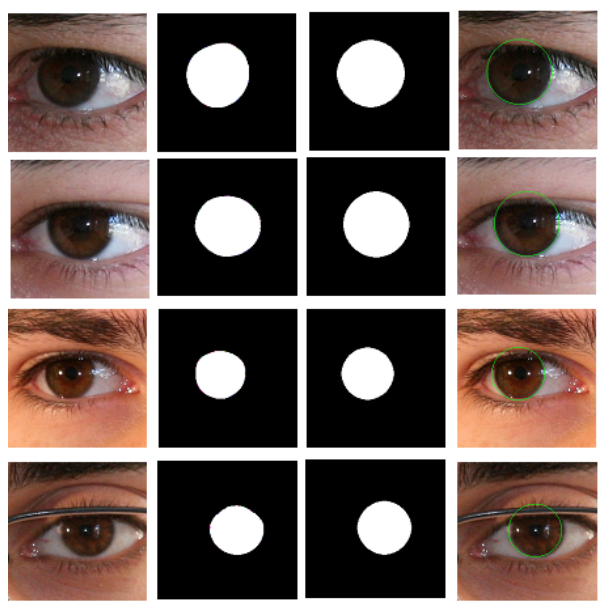

Fig. 8. Resultados do modelo de segmentação da íris

A tabela 1 apresenta os resultados da segmentação da íris por experimento, indicando o coeficiente de dice médio das imagens segmentadas, além de mostrar o desvio padrão desta medida de modo a compreender a contribuição do pósprocessamento com o Smallest Circle. Um experimento de ablação é realizada, removendo a etapa de ajuste da íris segmentada com o algoritmo Smallest Circle, a fim de verificar o desempenho da segmentação com e sem a aplicação do algoritmo.

O coeficiente de dice é calculado por imagem para a avaliação qualitativa e observação do comportamento na segmentação da íris, notando alguns pontos de possíveis melhorias em seus resultados. Em seguida calcula-se a média
TABLE I

Resultados da Rede Neural At-Unet

\begin{tabular}{|c|c|c|c|}
\hline Método & Database & $\begin{array}{c}\text { Desvio } \\
\text { Padrão }\end{array}$ & $\begin{array}{c}\text { Coeficiente } \\
\text { de Dice( \%) }\end{array}$ \\
\hline At-Unet + Módulo Atenção & MICHI-I & 0.0385 & 94.45 \\
(sem Smallest Circle) & UBIRIS.v2 & 0.0391 & 93.69 \\
\hline At-Unet + Módulo de Atenção & MICHI-I & 0.0241 & 95.32 \\
(com Smallest Circle) & UBIRIS.v2 & 0.0267 & 94.14 \\
\hline
\end{tabular}

de coeficiente de dice por base, para obter uma visão geral de desempenho desta metodologia. A partir disso os resultados iniciais conforme a tabela 1 são promissores, visto que ao realizar os experimentos desta metodologia, verifica-se que o desempenho da segmentação da íris se destaca ao aplicar o algoritmo Smallest Circle no pós-processamento.

Então a média de coeficiente de dice mínimo em imagens de testes ao aplicar o algoritmo Smalltest Circle por base é de 95.32\% na base MICHI-I e $94.14 \%$ na base UBIRIS.v2. Sem a aplicação do algoritmo a média da métrica em imagens de teste por base diminue em pelo menos $0.65 \%$, caracterizando que a etapa de pós-processamento é essencial para o bom desempenho da segmentação da íris, visto que as imagens dos olhos estão oclusas por cílios e pálpebras.

Um fato a destacar é que o desvio padrão diminue a cada experimento, constatando que o coeficiente de dice por imagem estão próximos da média por base, indicando também que, quanto menor o desvio padrão melhor o desempenho da segmentação. Durante os experimentos observou-se que algumas imagens segmentavam artefatos conforme citado na subseção 3.B. Em alguns testes qualitativos por imagem, existiam esssa pequenas áreas que representavam alguma parte da região dos olhos, como sobrancelhas e cílios. É importante destacar que a oclusão da íris em algumas imagens ocorriam devido ao uso de óculos e lentes.

De modo a situar a proposta no estado da arte atual, os resultados são comparados com trabalhos que realizam a segmentação da íris nas bases de dados UBIRIS.v2 e MICHII. A U-Net, FRED-Net [5], CNNHT [6] e Irispasenet [7] são métodos segmentação distintos, selecionados para avaliar o desempenho desta metodologia levando em consideração os dados de teste dessas bases.

A tabela 2 apresenta os resultados desta metodologia aplicada nas bases de testes dos autores citados para a comparação de resultados.

Em comparação com outros trabalhos de segmentação da íris conforme apresentado na tabela 2, a metodologia apresenta bons resultados. Assim como os resultados iniciais da tabela 1 , é calculado o coeficiente de dice médio por base de teste disponibilizada pelos autores a fim de validar a metodologia. O coeficiente de dice médio deste trabalho na base MICHI-I é 95.32\% e a base UBIRIS.v2 94.14\%, aproximadamente $3.85 \%$ maior que a proposta do CNNHT.

Ao comparar com a IrisParseNet identifica-se que os resultados estão bem próximos. Esta abordagem é aproximadamente $2.27 \%$ maior na média de coeficiente de dice quando comparado a base MICHI-I do o IrisParseNet, assim como 
TABLE II

COMPARAÇÃO DE DIFERENTES ABORDAGENS PARA A SEGMENTAÇÃO DA ÍRIS.

\begin{tabular}{|c|c|c|}
\hline Método & Database & $\begin{array}{c}\text { Coeficiente de Dice } \\
\mathbf{( \% )}\end{array}$ \\
\hline U-Net & MICHI-I & 90.25 \\
& UBIRIS.v2 & 91.77 \\
\hline CNNHT & MICHI-I & 91.41 \\
(Refinanet) & UBIRIS.v2 & 90.34 \\
\hline IrisParsenet & MICHI-I & 93.05 \\
(Multi-task/Attention) & UBIRIS.v2 & 91.78 \\
\hline & & \\
FRED-Net & MICHI-I & 93.15 \\
& UBIRIS & 93.84 \\
\hline At-Unet & MICHI-I & $\mathbf{9 5 . 3 2}$ \\
Com smallest Circle & UBIRIS.v2 & $\mathbf{9 4 . 1 4}$ \\
(Método proposto) & & \\
\hline
\end{tabular}

é aproximandamente 2,36\% maior na média de Dice quando relacionado a base UBIRIS.v2. Já analisando os resultados com o FRED-Net o coeficiente de dice médio é um pouco mais próximo dos resultados da metodologia proposta, apresentando uma diferença na média de dice de $2.17 \%$ na base MICHI-I e $0.99 \%$ na base UBIRIS.v2.

A segmentação baseada apenas na rede U-Net conforme apresentanda na tabela 2 , expõe resultados inferiores a metodologia deste trabalho, embora a arquitetura apresentar um bom desempenho na segmentação de imagens médicas, para a tarefa proposta neste trabalho são necessárias a inclusão de novas configurações e recursos na rede neural.

Os resultados do experimento três, a fim de avaliar a rede At-Unet, por meio da execução da validação cruzada, consta na tabela 3.

TABLE III

Resultados da Validação CruZada da Rede Neural At-Unet

\begin{tabular}{|c|c|c|}
\hline $\begin{array}{c}\text { k=5 } \\
\text { (Cinco Grupos de Treinamento) }\end{array}$ & Database & $\begin{array}{c}\text { Coeficiente de Dice } \\
(\mathbf{\%})\end{array}$ \\
\hline $\mathbf{k 1}$ & MICHI-I & 95.03 \\
& UBIRIS.v2 & 94.95 \\
\hline $\mathbf{k 2}$ & MICHI-I & 93.34 \\
& UBIRIS.v2 & 92.78 \\
\hline $\mathbf{k 3}$ & MICHI-I & 95.51 \\
& UBIRIS.v2 & 94.21 \\
\hline $\mathbf{k 4}$ & MICHI-I & 94.87 \\
& UBIRIS.v2 & 93.35 \\
\hline $\mathbf{k 5}$ & MICHI-I & 94.23 \\
& UBIRIS.v2 & 94.69 \\
\hline
\end{tabular}

Para a validação cruzada um conjunto de dados $k$, é dividido em cinco partes, ou seja $k=5$. Os grupos dos conjuntos de dados deste trabalho, são definidos por $k 1, k 2, k 3, k 4$ e $k 5$. Cada grupo é subdividido em treinamento, teste e validação, sendo assim os dados distribuídos aleatóriamente são diferentes para cada $k$. Desta forma foi possível avaliar o desempenho do modelo por todo os conjunto de dados.

Cinco rodadas de treino são executadas, no intuito de avaliar o desempenho do modelo durante o treinamento e teste. A média de dice da validação cruzada para a base de MICHI-I é de $94.59 \%$, e para a base UBIRIS.v2 é de $93,96 \%$.
O pior desempenho da rede neural segundo a tabela 3 , é identificada no grupo $k 2$, onde a média de coeficiente de dice é menor em relação aos outros grupos dos conjuntos de dados. No entanto, o desempenho do At-Unet é satifatória durante validação cruzada, pois apresenta uma média da métrica avaliada próxima de $95 \%$, indicando um bom desempenho desta metodologia para a tarefa proposta.

\section{Conclus Ão}

Este trabalho apresentou um método baseado em uma modificação da rede neural U-Net para a segmentação da íris em imagens coloridas do olho.

O método proposto neste trabalho é promissor por lidar com imagens oclusas por cílios, pálpebras e reflexos do ambiente refletidos na íris. Estas características indicam que o método pode ser utilizado com sucesso em arquiteturas de avaliação da íris e métodos para segmentação/detecção da pupila.

Destaca-se que a etapa de pós-processamento realizada com o algoritmo Smallest Circle contribui para um incremento dos resultados do método. Além disso a rede At-Unet colabora para o bom desempenho na tarefa de segmentação.

Como sugestão de trabalhos futuros indicamos a utilização dos resultados fornecidos por este método para segmentaçào da pupila possibilitando indentificar reações pupilares para avaliar os estados cognitivos de uma pessoa a ponto de diagnosticar algum distúrbio neurológico.

Está metodologia colabora para pesquisadores da visão computacional apresentado técnicas eficazes para a detecção da íris em tarefas de rastreamento ocular.

\section{AGRADECIMENTOS}

O presente trabalho foi realizado com apoio da Fundação de Amparo à Pesquisa e ao Desenvolvimento Científico e Tecnológico do Maranhão (FAPEMA), da Coordenação de Aperfeiçoamento de Pessoal de Nível Superior (CAPES) e do Conselho Nacional de Desenvolvimento Científico e Tecnológico (CNPq).

\section{REFERENCES}

[1] Min-Allah, N., Jan, F., Alrashed, S. Pupil detection schemes in human eye: a review. Multimedia Systems 27, 753-777 (2021).

[2] Gomai, A. El-Zaart and H. Mathkour, "A new approach for pupil detection in iris recognition system," 2010 2nd International Conference on Computer Engineering and Technology, 2010, pp. V4-415-V4-419.

[3] J. Daugman, "New Methods in Iris Recognition," in IEEE Transactions on Systems, Man, and Cybernetics, Part B (Cybernetics), vol. 37, no. 5, pp. 1167-1175, Oct. 2007.

[4] Liu, H. Li, M. Zhang, Jing Liu, Z. Sun and T. Tan, "Accurate iris segmentation in non-cooperative environments using fully convolutional networks," 2016 International Conference on Biometrics (ICB), 2016, pp. 1-8.

[5] Arsalan, Muhammad Kim, Dong Lee, Min Owais, Muhammad Kang, Ryoung. (2019). FRED-Net: Fully Residual Encoder-Decoder Network for Accurate Iris Segmentation. Expert Systems with Applications. 122. 10.1016/j.eswa.2019.01.010

[6] Hofbauer Heinz, Jalilian, Ehsaneddin, Uhl Andreas. (2018). Exploiting Superior CNN-based Iris Segmentation for Better Recognition Accuracy. Pattern Recognition Letters. 120. 10.1016/j.patrec.2018.12.021.

[7] C. Wang, J. Muhammad, Y. Wang, Z. He and Z. Sun, "Towards Complete and Accurate Iris Segmentation Using Deep Multi-Task Attention Network for Non-Cooperative Iris Recognition," in IEEE Transactions on Information Forensics and Security, vol. 15, pp. 2944-2959, 2020. 
[8] A. Elhassouny and F. Smarandache, "Trends in deep convolutional neural Networks architectures: a review," 2019 International Conference of Computer Science and Renewable Energies (ICCSRE), 2019, pp. 1-8.

[9] R. Chauhan, K. K. Ghanshala and R. C. Joshi, "Convolutional Neural Network (CNN) for Image Detection and Recognition," 2018 First International Conference on Secure Cyber Computing and Communication (ICSCCC), 2018, pp. 278-282.

[10] R.Olaf, F.Philipp, B.Thomas. (2015). U-Net: Convolutional Networks for Biomedical Image Segmentation.

[11] R. El Jurdi, C. Petitjean, P. Honeine and F. Abdallah, "BB-UNet: U-Net With Bounding Box Prior," in IEEE Journal of Selected Topics in Signal Processing, vol. 14, no. 6, pp. 1189-1198, Oct. 2020.

[12] W. Zhang, X. Lu, Y. Gu, Y. Liu, X. Meng, and J. Li, "A robust iris segmentation scheme based on improved U-Net.

[13] Simonyan, Karen Zisserman, Andrew. (2014). Very Deep Convolutional Networks for Large-Scale Image Recognition. arXiv 1409.1556.

[14] J. Deng, W. Dong, R. Socher, L. Li, Kai Li and Li Fei-Fei, "ImageNet: A large-scale hierarchical image database," 2009 IEEE Conference on Computer Vision and Pattern Recognition, 2009, pp. 248-255.

[15] Triyani, Yuli Nugroho, Hanung Adi Rahmawaty, Made Ardiyanto, Igi Choridah, Lina. (2016). Performance analysis of image segmentation for breast ultrasound images. 1-6.

[16] Li, Yung-Hui, Wenny R. Putri, Muhammad S. Aslam, and Ching-Chun Chang. 2021. "Robust Iris Segmentation Algorithm in Non-Cooperative Environments Using Interleaved Residual U-Net" Sensors 21, no. 4 1434. 